\title{
Article \\ High precision voltage measurement for optical quantum computation
}

\author{
Kamil Wereszczyński ${ }^{1 *}$,, Agnieszka Michalczuk ${ }^{1}{ }^{\circledR}$, Marcin Paszkuta $^{1}$ and Jacek Gumiela ${ }^{2,(}$ \\ 1 Department of Computer Graphics, Vision and Digital Systems \\ Faculty of Automatic Control, Electronics and Computer Science, Silesian University of Technology \\ Akademicka 16, 44-100 Gliwice, Poland \\ 2 Department of Electrical Power Engineering, Faculty of Electrical Engineering, Wroclaw University of \\ Science and Technology, Wybrzeze Wyspianskiego 27, 50-370 Wroclaw \\ * Correspondence: kamil.wereszczynski@polsl.pl; Tel.: (+48-726-562-182 (K.W.))
}

\begin{abstract}
We communicate the theoretical results in the study of utilizing the quantum phenomena in optical current for quantum computation in the context of high precision voltage measurements. These results can be used around computation by quantum sampling and quantum communication as the basis for further research and physical implementations. We propose a Main Optical Setup (MOS) for such computation which allows to make a superstructure to implement specific computations and algorithms. To create MOS we used the nonlinear units (e.g., beta- barium borate crystal), arranged in series, powered with pulsed laser pump, and ended with the beam splitter to generate the output state of a number of entangled photon pairs. The computation is made by propagation of entanglement with beam splitters applied crossword and adjustable phase shifters that are tools for parameter steering. We show how to implement the series of cosine-based components on the example of two-component case. The results opens a broad area for future research in the area of building quantum optimizer using the quantum sampling methods and in the area of high precision temporal measurement of voltage, which is the important process for building high-fidelity devices.
\end{abstract}

Keywords: quantum computing, quantum optics, quantum optimization, photon entanglement, coincidence measure, photodiodes current measure, voltage measurement

\section{Introduction}

Quantum phenomena arising in optical current can be utilized in quantum computation and quantum communication, specifically basing on those phenomena. There are many optical-based implementation of quantum computation task, e.g.: the KLM protocol proposed by Kneel et al. in [1], Photonic [2] or cat-qubits by Mirrahimi [3]. In this paper we propose a new concept of implementation quantum computation in optical current utilizing the distortion of homogeneous probability distribution over photo detectors, caused by phase shifts between rails. We show how to implement in optical current one component of Cosine series sampled operator (QCoSamp), that was described in our previous work: [4].

It is important, for all methods, the issue of measurement of the voltage caused by photo-diode sensors, that we use as the terminal of optical current. It can work in one of two modes: photovoltaic or photoconductive, however, due to necessity of, usually weak, signal amplification, at the measurement point, we obtain the voltage, and it is at the range of $[-3.5,3.5] \mathrm{mV}$, in our case.The mentioned protocols needs the high-precision (in time domain) voltage measurement e.g., KLM.

The three phenomena are indispensable for making the quantum computation and communication: superposition, interference and entanglement. However the superposition and interference arises in the streams of photons spontaneously, because of 
the nature of light (specifically the fact that photons "moves" with the speed of light), the entanglement does not appear in that simple way. It can be produced using SPDC (Spontaneous Parametric Down Conversion) process, described by Couteau in [5]. While the beam from laser pump goes through non-linear crystal, four times in a million, one photon is converted into two photons, which are correlated in time, certainly. Because of the energy conservation, the correlated photon pairs has doubled wavelength (or half frequency) as the original one and their paths are diverted. Depending on the crystal, there exists three types of SPDC: type 0, where the polarization of both photons are the same and same as the polarization of the input laser beam, type I, where the polarization of photons is still the same but orthogonal to the laser, and type II, where the polarization of the photons is orthogonal each other. We focused on the SPDC type II taking place in BBO ( $\beta$ barium borate) cristal, which has the additional property that the correlated pairs are diverged symmetrically. Therefore there arises two cones of light that intersect in two points, due to properly shaped geometry of the crystal. Historically, we name one of such a point "signal" and the second one "idler", however those two rays are nowadays combined to obtain greater beam intensity. Hence in the resulting ray, there appears time-correlated (which mean indistinguishable) photon pairs with cross - correlation. They are superposed as well, certainly. Therefore, after going through the beam splitter, we obtain two cross-polarized rays. Because the photons are superposed and time indistinguishable, they are entangled, which manifests itself in measuring the time correlation of set of photons (see Magnitskiy et al. in [6]). It was proven experimentally for ten photons entanglement by Wang et al. in [7] or for twelve by Zhong et al. in [8]. The prove lays on the detection of time coincidence of photons obtained in the photon detector. The laser beam is very narrow down to $10 \mathrm{fs}$ with up to $20 \mathrm{M}$ beams per second. The measurement is made by photon counters ending each ray, and the Correlation Counter, which returns the number of photons which appears in both detectors in the same time interval of the 81ps length.

In this context we communicate the idea how to implement the QCoSamp component. It was described in our previous work [4], leads to obtain the function $\frac{1}{2}(1-\cos (x))$. In this work, the changes of the variable $x$ are achieved by changing the phase in phase gate $P_{\varphi}$. Hence, the idea is to prepare the quantum state in such a way that with changing the parameter $\varphi$ we obtain the desired value. If we dispose such a component we can add a number of them to generate the series. We proved (ibidem) that such a series maps into Fourier series. Therefore using it together with other quantum techniques, like distributed phase encoding (Ruiz-Perez et al. in [9]) and amplitude amplification (see $[10,11]$ ) we can create a number of algorithms, e.g., curve fitting, and even signal and image extraction.

\section{Materials and methods}

In this section we present the quantum description of optical phenomena used in quantum computation. Then we present selected protocols of quantum computation. Finally we present the optical setup that was used in our experiments.

\subsection{Quantum description of optical phenomena}

The quantum mechanical description of optical elements is based on two concepts. Firstly, we will use the Fock space, which describes the quantum state made of many photons. Consider, that eigen basis of the photons consists of $k$ eigen states (e.g. polarization). The basis state of the whole system of $n$ photons is the sequence of numbers $\phi_{j}$ being cardinalities of photons which are in the state $j$. The states are called modes; the state $|0\rangle=|0 \ldots 0\rangle$ is called vacuum. Secondly we define the annihilation $\hat{a}$ and creation $\hat{a}^{\dagger}$ operators as follows: $\hat{a}|0\rangle=0, \hat{a}|n\rangle=\sqrt{n}|n-1\rangle, \hat{a}^{\dagger}|n\rangle=\sqrt{n+1}|n+1\rangle$. The Lie algebra of such operators is given by commutators: $\left[\hat{a}_{k}, \hat{a}_{m}^{\dagger}\right]=\delta(k-m),\left[\hat{a}_{k}, \hat{a}_{m}\right]=\left[\hat{a}_{k}^{\dagger}, \hat{a}_{m}^{\dagger}\right]=0$ With such tools we can define optical devices (after Kok et al. [12]) by defining the number of inputs and outputs, set of parameters, and evolution operator acting on the 
photons going through the device. Those operators will be defined as a set of creation operators for each output separately, depending on parameters and creation operators for inputs.

The simplest optical device is the single-mode phase shifter (PS) changing the phase of incoming photons by phase $\varphi$ that has one input $S_{i}$ and one output $S_{o}$. Its evolution operator is defined as follows:

$$
\hat{S}_{o}^{+}=e^{i \varphi} \hat{S}_{i}^{\dagger}
$$

Beam splitter (BS) or Polarization rotator has two inputs $B_{i 1}, B_{i 2}$, two outputs $B_{01}, B_{02}$ and is parametrized by $R=\cos ^{2} \theta$ reflection rate and $T=1-R=\sin ^{2} \theta$ transmission rate. Furthermore we assume that the incoming waves can differ by the phase $\varphi$. In that case:

$$
\begin{aligned}
& \hat{B}_{o 1}^{\dagger}=\cos \theta \hat{B}_{i 1}^{+}+i e^{-i \varphi} \sin \theta B_{i 2}^{\dagger} \\
& \hat{B}_{o 2}^{+}=i e^{i \varphi} \sin \theta B_{i 1}^{\dagger}+\cos \theta \hat{B}_{i 2}^{\dagger}
\end{aligned}
$$

Qubit is represented by element of $S U(2)$. The photon-based implementation of such a group can be made using the photon polarization creating polarization qubits. It can be utilized in two ways: one-rail, where photons with different polarization exists in one ray, called spatial mode; dual-rail, where there are two spatial modes - one for each orthogonal polarization. The modes can be easily switched using beam splitter and phase rotators. Let's assume that vertically polarized qubit will be denoted $|V\rangle$ and horizontally - $|H\rangle$ in Hilbert space. The Fock state will have two slots - one for vertical and one for horizontal polarization: $|1,0\rangle=|H\rangle$ and $|0,1\rangle=|V\rangle$. The general state of one qubit is defined as follows:

$$
|q\rangle=a|V\rangle+b|H\rangle=a|0,1\rangle_{F}+b|1,0\rangle_{F}
$$

Let's assume, that on the input of beam splitter, there appears $n$ photons to the $o 1$ input and $m$ photons to the $o 2$ input. We can formally denote it with annihilation and creation operator in Fock space: $|n, m\rangle_{F}=\left[\hat{B}_{i 1}^{\dagger}\right]^{n}\left[\hat{B}_{i 2}^{\dagger}\right]^{m}|0\rangle_{F}$. After coming through the beam splitter this input operators will change to the output operator that transforms according to the eq. 2. Using this transformation (and Pythagorean identity) we can write the input operator in therms of output operators, as follows:

$$
\begin{aligned}
& \hat{B}_{i 1}^{+}=\cos \theta \hat{B}_{o 1}^{+}-i e^{-i \varphi} \sin \theta \hat{B}_{o 2}^{+} \\
& \hat{B}_{i 2}^{+}=\cos \theta \hat{B}_{o 2}^{+}-i e^{i \varphi} \sin \theta \hat{B}_{o 1}^{+}
\end{aligned}
$$

Hence, the output state of the beam splitter for an input $|n, m\rangle_{F}$ is equal to:

$$
\left[\cos \theta \hat{B}_{o 1}^{\dagger}-i e^{-i \varphi} \sin \theta \hat{B}_{o 2}^{+}\right]^{n}\left[\cos \theta \hat{B}_{o 2}^{+}-i e^{i \varphi} \sin \theta \hat{B}_{o 1}^{+}\right]^{m}|0\rangle_{F}
$$

\subsection{KLM protocol}

Let's consider the system of two qubits, one polarized vertically and one horizontally and let them pass through the beam splitter. Using creation operator notation, on the output of the beam splitter we obtain both output operators acting on the vacuum: $|x, y\rangle_{F}=\hat{B}_{o 1}^{\dagger} \hat{B}_{o 2}^{+}|0\rangle$, where index $F$ means that we consider the Fock state. Now, applying eq. 5 and remembering that commutator for two creation operators zeroes, we obtain: 


$$
\begin{aligned}
& |1,1\rangle_{F}=\hat{B}_{i 1}^{+} \hat{B}_{i 2}^{+}|0\rangle_{F} \stackrel{\mathrm{BS}}{\longrightarrow} \\
& {\left[\cos (\theta) \hat{B}_{o 1}^{\dagger}-i e^{-i \varphi} \sin (\theta) \hat{B}_{o 2}^{\dagger}\right]\left[\cos (\theta) \hat{B}_{o 2}^{\dagger}-i e^{i \varphi} \sin (\theta) \hat{B}_{o 1}^{\dagger}\right]|0\rangle_{F}=} \\
& {\left[\cos (2 \theta) \hat{B}_{o 1}^{\dagger} \hat{B}_{o 2}^{\dagger}-\frac{i}{2} \sin (2 \theta)\left(e^{i \varphi}\left[\hat{B}_{o 1}^{\dagger}\right]^{2}+e^{-i \varphi}\left[\hat{B}_{o 2}^{+}\right]^{2}\right]|0\rangle_{F}=\right.} \\
& \cos (2 \theta)|1,1\rangle_{F}-\frac{1}{\sqrt{2}} i \sin (2 \theta)\left(e^{i \varphi}|2,0\rangle_{F}+e^{-i \varphi}|0,2\rangle_{F}\right)
\end{aligned}
$$

If we consider BS with $R=T=1 / 2$, (hence $\theta=\pi / 4$ ) and phase $\varphi=\pi / 2$ then $\sin (2 \theta)=1, \cos (2 \theta)=0$ and $e^{i \pi / 2}=-1, e^{-i \pi / 2}=1$ and the system is described as follows:

$$
\frac{1}{\sqrt{2}}\left(|0,2\rangle_{F}-|2,0\rangle_{F}\right)
$$

The above equation means the absence of the output state $|1,1\rangle$, which means that after passing through beam splitter both photons has the same polarity. This is completely quantum effect, because classically nothing shall prevent the pair of cross-polarized photons to have orthogonal polarizations after passing the beam splitter ${ }^{1}$. Photons pair off together has a name of photon bunching. Complementary, the absence of $|1,1\rangle$ state has the name Hong-Ou-Mandel effect described in [13]. Photon bunching is one of the pillars of the KLM (Knil et al., ibidem) scheme and its extensions.

The second one is nonlinear sign (NS) gate, which is the building block of other KLM gates. Let's consider a state $|\psi\rangle=a|0\rangle+b|1\rangle+c|2\rangle$ in Fock space. The NS gate acts by changing the last coordinate $c$ to $-c$. For example of using those two notions let us to construct the simple two-qubit controlled $\mathrm{Z}$ gate, that flips the sign of $|H\rangle \otimes|H\rangle=|1,0,1,0\rangle$ coordinate, while the others remain unchanged. Thus let's consider two-qubit, separable state $|x\rangle \otimes|y\rangle=(\alpha|0,1\rangle+\beta|1,0\rangle)(\gamma|0,1\rangle+\psi|1,0\rangle)$ The CZ gate should change the $\beta \psi$ to $-\beta \psi$ so the application of NS gate is tempting, however in the last state there are no two photons in the same mode. But if we connect first $(\alpha|0,1\rangle)$ and third $\gamma|0,1\rangle)$ with beam splitter, the photon bunching arise (see eq. 7 ) and on output of beam splitter are the state, which can be changed by NS applied to the same modes. Finally we act on the same modes with beam splitter once again and we obtain the final state equal to $C Z|x\rangle \otimes|y\rangle$.

Unfortunately, the NS gate cannot be constructed using linear optics element. The only thing we can do is to create a gate that will work like CZ gate with the given probability of success and indicates the proper result with an additional outputs. Therefore such gates name is probabilistic gates. Knill in [14] proved that the upper bound of such a probability is equal to $1 / 2$. The physical implementations probabilities are in the range 0.075 to 0.22 - see Kok et al. (ibidem) for details. The fidelity of those implementation is $80 \%$ (e.g. $79 \%$ by O'Brian or $84 \%$ by Gasparoni et al. $)^{2}$

One of the most major reason for this relatively low fidelity is necessity of measure the correlation between the output and the indicator of correctness. Both the result and the indicator are measured using photo detectors, which uses photo-voltaic effect to build up the voltage proportional to the energy of incoming light. Since the wavelength of the source is known the photon count can be computed basing on the output voltage.

\subsection{Measurement setup}

The measurement setup consists of: (1) photon-to-voltage converters, based on photo-diodes, (2) amplifiers (optionally) and (3) coincidence counter. Photon-to-voltage can work in one or both of tribes: photovoltaic or photoconductive. The first one gives more robust results but is slightly slower, the second one creates the dark current and

\footnotetext{
1 Moreover the state $|1,1\rangle$, classically, has probability $1 / 2$, because it covers two states: $|V\rangle \otimes|H\rangle$ and $|H\rangle \otimes|V\rangle$ that are indistinguishable.
}

We don't put here references due to lack of place. It can be found in Kok et al. (ibidem) review. 


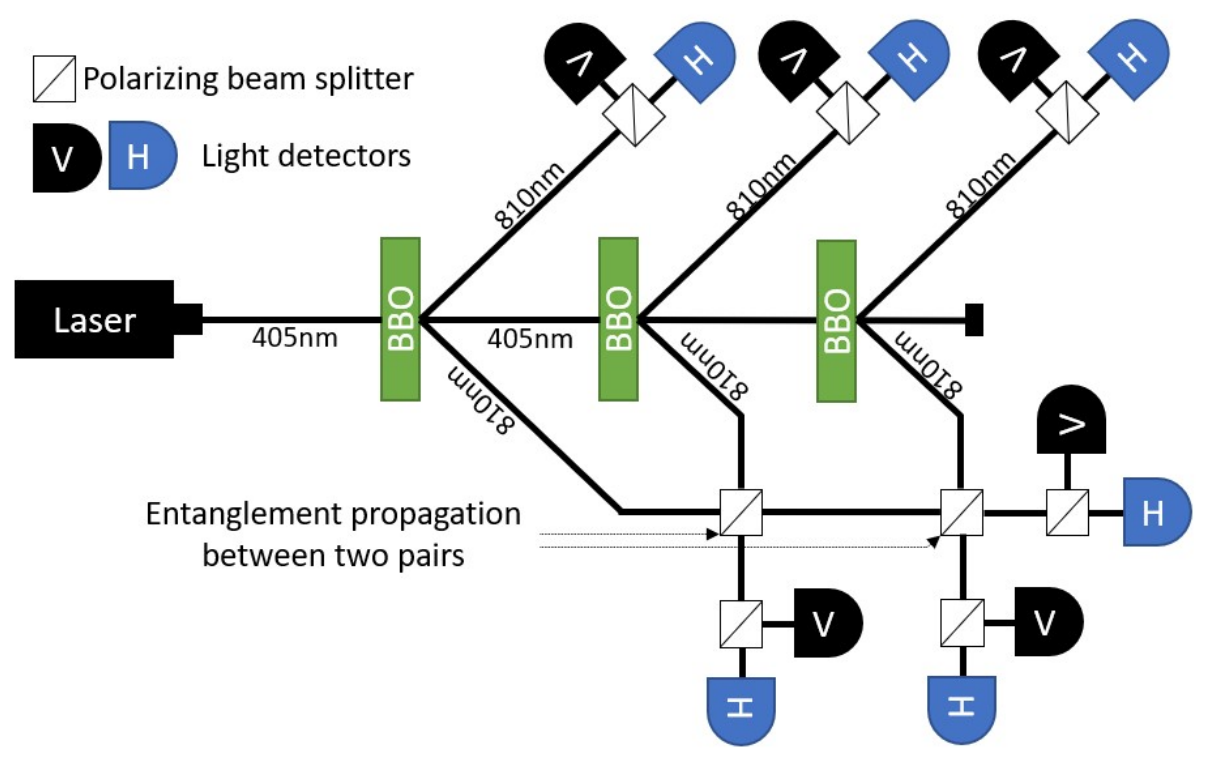

Figure 1. 6 photon entanglement setup. Firstly, the two pairs of photons are generated by nonlinear unit in series, here BBO crystal. Then one photon from each pair is connected with the remaining part of the system using polarization beam splitter. The connection points are places, where entanglement propagates trough the whole system and creates the GHZ states, finally.

there arises the Johnson-Nyquist noise sometimes, hence is faster. The avalanche photodiodes, working in Geiger mode can notice separate photons, however they need high voltage and has relatively low level of saturation, therefore they are not proper for the beams with high energy.

Existing photon-to-voltage converters has relatively long rise/fall time. For example: the silicon continuous photo-diode from Thorlabs SM05PD2A has this parameter equal to $1 \mathrm{~ns}$, the maximum reverse - bias voltage $25 \mathrm{~V}$ and quantum efficiency on the level of 0.41; Indium gallium arsenide (InGaAs) biased, high speed detector DET08CL/M has $70 \mathrm{ps} / 110 \mathrm{ps}$ rise/fall, $22 \mathrm{~V}$ bias, $5 \mathrm{GHz}$ measurement frequency and quantum efficiency 0.47 . In the area of coincidence counter the solution widely used is ID Quantique 800 from Photonics, which has coincidence time-interval equal to $81 \mathrm{ps}$ and $2 \mathrm{MHz}$ frequency, ID 900 time controller with precision 20ps with possible 20M measurement cycles per second.

\section{Results}

In our previous work, we defined theoretically the QCoSamp method of quantum computation [4], basing on the quantum sampling, which creates the probability distribution over the measurement basis. The results are interpreted in the context of trigonometric series made of components: $\frac{1}{2}(1+\cos (n x+r))$. The light creates a probability distribution over the detectors, that could be recognized as the measurement basis of the optical quantum system. On the other hand, the state of the photon on the output of the BBO crystal in SPDC phenomenon is equal to (after Magnitskiy et al., ibidem, pg. 620):

$$
\frac{|H V\rangle+e^{i \delta}|V H\rangle}{\sqrt{2}}
$$



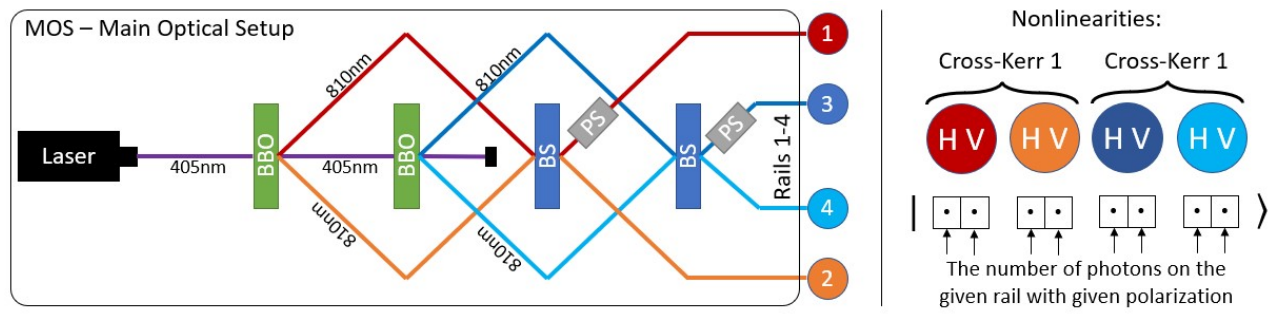

Figure 2. The main optical setup (MOS) of the system (left side) and the Fock state meaning (right side). BBO is a crystal realizing the SPDC, BS-Beam Splitter and PS - phase shifter. The signal and output rails from $\mathrm{BBOs}$ are marked by colors: red and orange (first $\mathrm{BBO}$ ) and dark and light blue (second $\mathrm{BBO}$ ). The lines crossing on the scheme should not cross in real setup. The rails beginning in two BBOs $(1,2)$ and $(3,4)$ contains the stream of entangled photon pairs in a state described by Magnitskiy et al., ibidem : $\frac{|H V\rangle+e^{i \delta}|V H\rangle}{\sqrt{2}}$. Then they goes through the $50 \% / 50 \%$ beam splitters (pairs from each BBO separately). The circles outside the area of MOS symbolize its 4 output rails. Because of Hong-Ou-Mandel effect they groups in one of beam splitter outputs, so on the output we obtain $|20\rangle$ or $|02\rangle$ states. The final state is the tensor product of those two, so we obtain one of 4 states $|2000\rangle,|0200\rangle,|0020\rangle,|0002\rangle$. Nevertheless, this description of state is not sufficient, since the cross-Kerr non-linearities generates the entanglement in the polarization domain, which is not covered in this description. Therefore, we propose the notation of state visualized on the right side of figure above, where for each mode there are two slots in the ket: first for horizontal polarization and second for vertical one. This notation will be called rail-polarization $(R P)$ notation.

Wang et al. [7] and Zhong et al. [8] uses SPDC with more sophisticated nonlinear units for generation of 10 and 12 photons. They uses the polarization beam splitter to obtain so called Greenberger-Horne-Zeilinger (GHZ) state for $n$ photons:

$$
\left|G H Z_{n}\right\rangle=\frac{1}{\sqrt{2}}\left(|H\rangle^{\otimes n}+|V\rangle^{\otimes n}\right)
$$

The technique (see fig. 1 involves to create $\frac{n}{2}$ pairs of photons using nonlinear unit (e.g., $\mathrm{BBO})$ in series. Then the entanglement is propagated by $\frac{n}{2}$ polarizing beam splitters. Each of them connects the existing entanglement with the next pair generated in the line. In our work, we use similar setup, but for computation we need more states then just GHZ, therefore, we connect both sides of the outputs from non-linear unit and we use non-polarizing beam splitter, which creates together very interesting states, from quantum computation perspective.

\subsection{The optical setup for QCoSamp implementation}

Let's apply the 50:50 beam splitter on this state for obtaining the two building blocks of the optical setup (see fig. 2). Since the input state is $|1,1\rangle_{F}$, we will use eq. 4. The parameter $\theta$ is still equal to $\pi / 4$, so $\cos (2 \theta)=0, \sin (2 \theta)=1$. We assume here for simplicity that the phase $\delta$ is equal to zero, nonetheless we have to remember, that without any involvement, this will not be a case. how to achieve it will be described later during defining the setup calibration process. The Magnitskiy equation 8 in the rail-polarization (RP) notation (see right part of fig. 2) will be denoted ${ }^{3}$ :

$$
\frac{1}{\sqrt{2}}(|10,01\rangle+|01,10\rangle) \text {. }
$$

\footnotetext{
3 For one BBO we have two rails, so RP state will have four slots, we omit, for simplicity, the vacuum state of the remaining rail, since it is not involved in the process.
} 


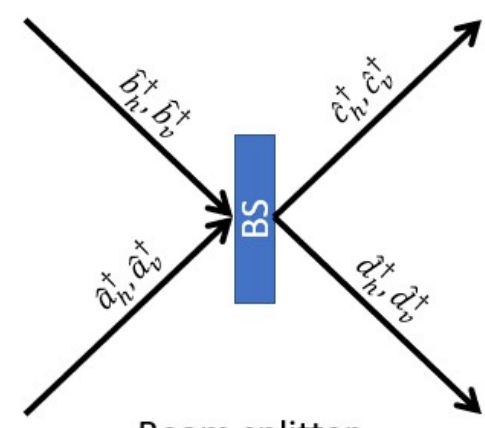

Beam splitter

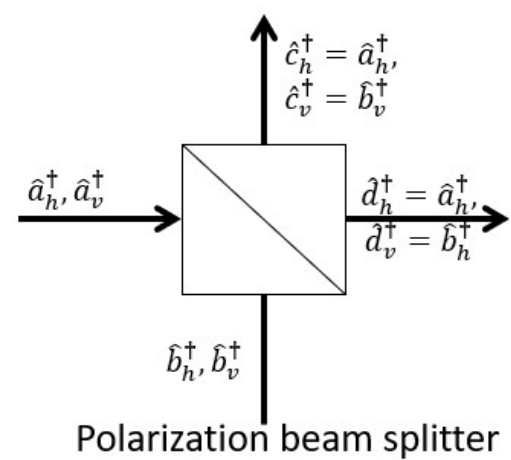

Polarization beam splitter

Figure 3. Beam splitter (left side) and polarization beam splitter (right side) acting on the two-rail states with polarization involved.

Moreover, we will denote the creation operators for the first input of a beam splitter with $\hat{a}_{h}^{\dagger}, \hat{a}_{v}^{\dagger}$, respectively for horizontal and vertical polarized photons, $\hat{b}_{h}^{+}, \hat{b}_{v}^{+}$for the second input, $\hat{c}_{h}^{\dagger}, \hat{c}_{v}^{\dagger}$ for the first output and $\hat{d}_{h}^{\dagger}, \hat{d}_{v}^{\dagger}$ for the second output, like on the fig. 3.

Here we will provide a list of two and four creation operators, that will be useful in the remaining part of this work. Right now we need just two of them (namely: $\hat{a}_{h}^{+} \hat{b}_{v}^{+}+\hat{a}_{v}^{+} \hat{b}_{h}^{+}$), but it is convenient to have them in one place. We don't provide proofs because one can easily derive them using the fact that creation operators commutes each other and expand the formula 5 . We assume that the BS is $50 \%-50 \%$ and on the one output it has the phase shifter with the relative phase $\varphi$. The list is as follows:

$$
\begin{aligned}
& \hat{a}_{h}^{\dagger} \hat{b}_{v}^{\dagger}=-\frac{1}{2} i\left(e^{i \varphi} \hat{c}_{h}^{\dagger} \hat{c}_{v}^{\dagger}+e^{-i \varphi} \hat{d}_{h}^{\dagger} \hat{d}_{v}^{\dagger}\right) \\
& \hat{a}_{v}^{\dagger} \hat{b}_{h}^{+}=-\frac{1}{2} i\left(e^{i \varphi} \hat{c}_{h}^{\dagger} \hat{c}_{v}^{\dagger}+e^{-i \varphi} \hat{d}_{h}^{\dagger} \hat{d}_{v}^{\dagger}\right) \\
& \hat{a}_{h}^{\dagger} \hat{b}_{h}^{\dagger}=-\frac{1}{2} i\left(e^{i \varphi}\left[\hat{c}_{h}^{\dagger}\right]^{2}+e^{-i \varphi}\left[\hat{d}_{h}^{\dagger}\right]^{2}\right) \\
& \hat{a}_{v}^{\dagger} \hat{b}_{v}^{+}=-\frac{1}{2} i\left(e^{i \varphi}\left[\hat{c}_{v}^{\dagger}\right]^{2}+e^{-i \varphi}\left[\hat{d}_{v}^{\dagger}\right]^{2}\right) \\
& \hat{a}_{h}^{\dagger} \hat{a}_{v}^{\dagger}=\frac{1}{2}\left(\hat{c}_{h}^{\dagger} \hat{c}_{v}^{\dagger}-i e^{-i \varphi} \hat{c}_{h}^{\dagger} \hat{d}_{v}^{\dagger}-i e^{-i \varphi} \hat{c}_{v}^{\dagger} \hat{d}_{h}^{\dagger}-e^{-i 2 \varphi} \hat{d}_{h}^{\dagger} \hat{d}_{v}^{\dagger}\right) \\
& \hat{b}_{h}^{+} \hat{b}_{v}^{+}=\frac{1}{2}\left(\hat{d}_{h}^{\dagger} \hat{d}_{v}^{+}-i e^{-i \varphi} \hat{c}_{h}^{\dagger} \hat{d}_{v}^{\dagger}-i e^{-i \varphi} \hat{c}_{v}^{\dagger} \hat{d}_{h}^{+}-e^{-i 2 \varphi} \hat{c}_{h}^{\dagger} \hat{c}_{v}^{\dagger}\right) \\
& \hat{a}_{h}^{\dagger} \hat{a}_{v}^{\dagger} \hat{b}_{h}^{+} \hat{b}_{v}^{\dagger}=-\frac{1}{4}\left(e^{i 2 \varphi}\left[\hat{c}_{h}^{\dagger}\right]^{2}\left[\hat{c}_{v}^{\dagger}\right]^{2}+\left[\hat{c}_{h}^{\dagger}\right]^{2}\left[\hat{d}_{v}^{\dagger}\right]^{2}+\left[\hat{c}_{v}^{\dagger}\right]^{2}\left[\hat{d}_{h}^{\dagger}\right]^{2}+e^{-i 2 \varphi}\left[\hat{d}_{h}^{\dagger}\right]^{2}\left[\hat{d}_{v}^{\dagger}\right]^{2}\right)
\end{aligned}
$$


To have all our puzzles together, we create the list describing the transformations of states needed in our computation model, twin to aforementioned list of operators, as follows:

$$
\begin{aligned}
& |10,01\rangle \stackrel{\mathrm{BS}}{\rightarrow}-\frac{1}{2} i\left(e^{i \varphi}|11,00\rangle+e^{-i \varphi}|00,11\rangle\right) \\
& |01,10\rangle \stackrel{\mathrm{BS}}{\rightarrow}-\frac{1}{2} i\left(e^{i \varphi}|11,00\rangle+e^{-i \varphi}|00,11\rangle\right) \\
& |10,10\rangle \stackrel{\mathrm{BS}}{\rightarrow}-\frac{1}{2} i\left(e^{i \varphi}|20,00\rangle+e^{-i \varphi}|00,20\rangle\right) \\
& |01,01\rangle \stackrel{\mathrm{BS}}{\rightarrow}-\frac{1}{2} i\left(e^{i \varphi}|02,00\rangle+e^{-i \varphi}|00,02\rangle\right) \\
& |11,00\rangle \stackrel{\mathrm{BS}}{\rightarrow} \frac{1}{2}\left(|11,00\rangle-i e^{-i \varphi}|01,10\rangle-i e^{-i \varphi}|10,01\rangle-e^{-i 2 \varphi}|00,11\rangle\right) \\
& |00,11\rangle \stackrel{\mathrm{BS}}{\rightarrow} \frac{1}{2}\left(|00,11\rangle-i e^{-i \varphi}|01,10\rangle-i e^{-i \varphi}|10,01\rangle-e^{-i 2 \varphi}|11,00\rangle\right) \\
& |11,11\rangle \stackrel{\mathrm{BS}}{\rightarrow}-\frac{1}{4}\left(|20,02\rangle+|02,20\rangle+e^{i 2 \varphi}|22,00\rangle+e^{i 2 \varphi}|00,22\rangle\right)
\end{aligned}
$$

In the virtue of above, the acting of BS (50\%-50\%, $\varphi=0)$ on the state on the output of BBO crystal is as follows:

$$
\frac{1}{\sqrt{2}}(|10,01\rangle+|01,10\rangle) \stackrel{\mathrm{BS}}{\longrightarrow}\left[\frac{1}{\sqrt{2}}\left(\hat{a}_{h}^{\dagger} \hat{b}_{v}^{\dagger}+\hat{a}_{v}^{\dagger} \hat{b}_{h}^{\dagger}\right)\right]|0\rangle=-\frac{1}{\sqrt{2}} i(|11,00\rangle+|00,11\rangle)=|\Psi\rangle
$$

The above equation means, that while two entangled photons just after leaving the nonlinear unit are not in the same rail, after crossing in beam splitter are both in "left" or „right' rail. Nevertheless the original state (eq. 8) doesn't change at all.

MSO (see fig. 2) consists of two such operations, therefore at the output there are four rails and four photons, entangled in pairs, for now. Therefore the final output state $|M S O\rangle$ is a tensor product of two states $\left|\Psi_{1}\right\rangle$ for the first nonlinear unit and $\left|\Psi_{2}\right\rangle$ for the second one. Therefore, we can write the final state of MSO as follows:

$$
\begin{aligned}
|M S O\rangle & =\left|\Psi_{1}\right\rangle \otimes\left|\Psi_{2}\right\rangle= \\
& =-\frac{1}{2}(|11,00 ; 11,00\rangle+|11,00 ; 0011\rangle+|00,11 ; 11,00\rangle+|00,11 ; 00,11\rangle)
\end{aligned}
$$

We have so far assumed that the relative phases of the light in both nonlinear units and beam splitters are equal to zero. However it not achievable situation in practical implementations. Therefore, we have to compensate the phases arising using the adjustable phase shifters placed on the one of the output of both beam splitters as is shown of fig. 2. The adjustable phase shifter is implemented by electro-optic phase modulator that shifts the phase of light in the range $[-\pi, \pi]$. The shift is a linear function of applied voltage. The process of such a compensation we will call the setup calibration. Because, so far, the optical currents for each nonlinear unit are separated, the calibration can be done for each of them separately. The problem is that the relative phase is not measurable directly - both states in eq. 13 has the same probability of obtaining it in measurement. Therefore we have to use the Mach-Zehnder interferometer [15-17], which help us to extract the phase. Hence the procedure of calibration can be done in two steps:

- Extend the optical current for the given nonlinear unit by Mach-Zehnder interferometer.

- $\quad$ Change the phase of light using the adjustable phase-shifter until the both output from the interferometer will have the same intensity. 


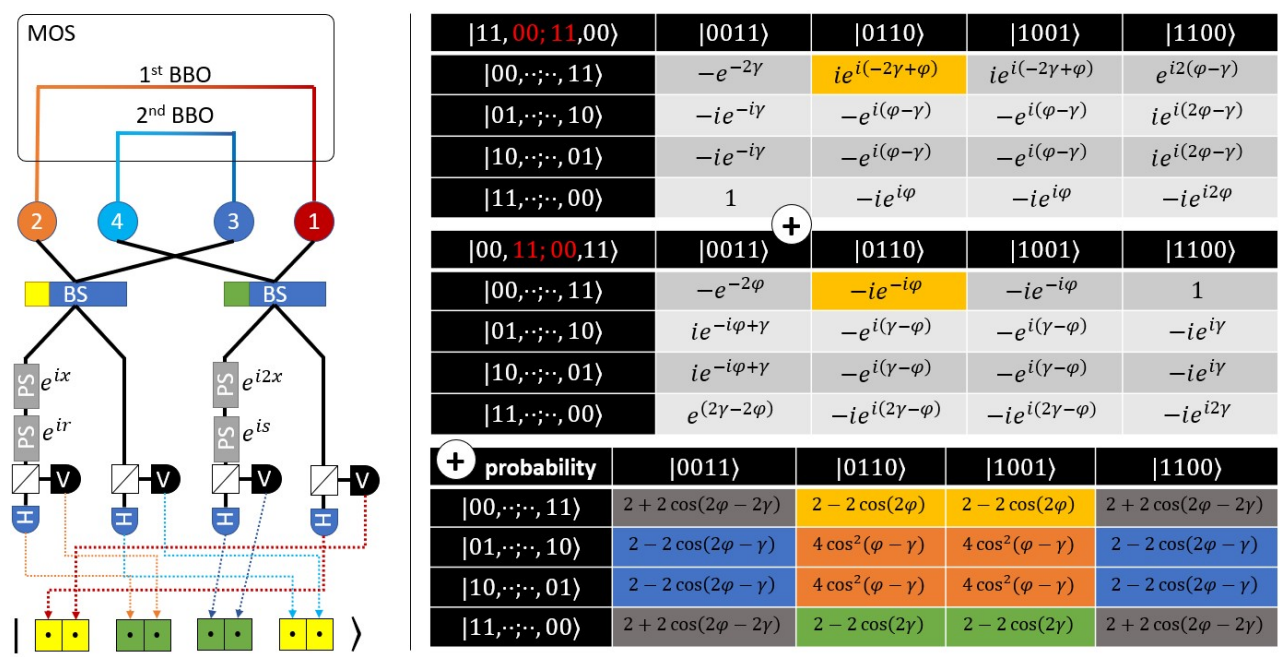

Figure 4. The scheme of the optical current (left side) and the tables with coefficients for two states after applying this current.

The calibration has to be done every time the setup will be changed and after a long period of time since the last one.

\subsection{QCoSamp component implementation using MSO}

In this section we present the general method of QCoSamp Component implementation and their addition. The optical current that implements two components and add them is presented on the fig 4 . It starts with pre-prepared and calibrated MOS, which has four output rails. Its state is given by eq. 14 . The rails: one and two contains entangled photon pairs and the other pair exists on the rails three and four. Hence we have two entangled system separated. Now, we propagate the entanglement (using the method of Wang [7] and Zhong [8]) to the whole system using two beam splitters, marked on that figure with yellow and green. On the one of outputs of beam splitters there is a phase shifter which serve us as the tool for introduce the parameter of the components: $\varphi, \gamma$. Then the rails, where exists horizontal and vertical polarized photon are divided with polarizing beam splitter. After that on the separate lines there are photon with the same polarization. Afterwards we measure those lines with photo detectors to obtain the state in a way shown on the bottom of the scheme. This procedure leads, in the context of state notation in Fock space to the connection of two middle pairs (marked with green color on the scheme) and two outboard pairs.

The acting of beam splitter on two pairs out of four leaves the remaining part of the state untouched. For example, let's consider we act on the middle two pairs of the state $|11,00 ; 11,00\rangle$, which gives us "middle" state: $|\cdot, 00 ; 11, \cdot\rangle$. We use the list of state transformation by beam splitter with relative phase (eq. 12) for the state $|00,11\rangle$ hence we obtain:

$$
|\cdot, 00 ; 11, \cdot\rangle \stackrel{\mathrm{BS}}{\longrightarrow} \frac{1}{2}\left(|\cdot, 00 ; 11, \cdot\rangle-i e^{-i \varphi}|\cdot, 01 ; 10, \cdot\rangle-i e^{-i \varphi}|\cdot, 10 ; 01, \cdot\rangle-e^{-i 2 \varphi}|\cdot, 11 ; 00, \cdot\rangle\right)
$$

On the other hand, if we consider the acting of the second beam splitter we obtain the "outboard" input state: $|11, \cdot ;, 00\rangle$, and the output will be as follows:

$$
|11, \cdot ; \cdot, 00\rangle \stackrel{\mathrm{BS}}{\longrightarrow} \frac{1}{2}\left(|11, \cdot ; \cdot, 00\rangle-i e^{-i \varphi}|01, \cdot ; \cdot, 10\rangle-i e^{-i \varphi}|10, \cdot ; \cdot, 01\rangle-e^{-i 2 \varphi}|00, \cdot ; \cdot, 11\rangle\right)
$$

Connecting those two states together, we have to make everyone-to-everyone connection of both output states where coefficients has to be multiplied, which is shown on the fig. 4 on the right-top table. The right-middle table shows the same operation for the 
state $|00,11 ; 00,11\rangle$. As one can easily see those two input states $(|11,00 ; 11,00\rangle$ and $|00,11 ; 00,11\rangle$ produces the same output state list but with different coefficients which are the functions of relative phases: $\varphi, \gamma$. This is the crucial observation, because the coefficient for the same output state will add, hence measuring of the state will extract the differences of the phases, just like last Hadamard operation in QCoSamp. For example the state $|00,01 ; 10,11\rangle$ (cell $(1,2)$ in mentioned tables, highlighted with yellow color), will have the coefficient: $i e^{i(-2 \gamma+\varphi)}-i e^{-i \varphi}=i e^{-i \varphi}\left(e^{-i 2 \varphi}-1\right)$.. The probability ${ }^{4}$ of obtaining this state will be equal to: $i e^{-i \varphi}\left(e^{-i 2 \varphi}-1\right) \cdot-i e^{i \varphi}\left(e^{i 2 \varphi}-1\right)=2-2 \cos (2 \varphi)$. So if we assume that $x=2 \varphi$ we obtain the value of the QCoSamp component.

On the mentioned figure in right-bottom table there are listed all probabilities ${ }^{5}$ that we obtain the state relative to given cell. We can see there that our setup in fact encodes five functions because the values repeats. One can extract any of such function and its sums, but for us the important cells are marked by yellow and green color.

The remaining two states $(|11,00 ; 00,11\rangle,|00,11 ; 11,00\rangle$ will transform as follows:

$$
|11,11\rangle \stackrel{\mathrm{BS}}{\longrightarrow}-\frac{1}{4}\left(|20,02\rangle+|02,20\rangle+e^{i 2 \varphi}|22,00\rangle+e^{i 2 \varphi}|00,22\rangle\right) .
$$

They will not add-up because they will obtain 2 in the crossed sections. Therefore, we cannot obtain the phases - the probability of obtaining them will be equal to $\frac{1}{16}$. Since there are 8 such states they will take $\frac{1}{2}$ of the probability. We can omit them in our considerations and treat as a noise.

The last question in out computation model is, how, in fact, is a qubit represented in our system. We can say that each pair (polarization, rail) represents qubit. However, there are two problems with such representation - we have to measure each photon separately, and there will be a huge number of photons that don't represent qubits. Moreover there is no way to distinguish which photons are qubits and which not observing just one photon. It can be done by observing temporal coincidence of four photons. The correlated fours points the photon being qubits. Because of that, the other approach is more natural. We can use 4 dimension qudits, instead of qubit. We will call them ququartits ${ }^{6}$. The ququartit is represented by a number of time correlated photons in one rail. Therefore one ququartit can have one of values: $|00\rangle,|01\rangle,|10\rangle,|11\rangle$ or in short: $|0\rangle,|1\rangle,|2\rangle,|3\rangle$.

In above representation we can observe just first and second ququartit. We sum-up the probability distribution obtained for this ququartits for the basis states: $|01\rangle,|02\rangle$ for the parameter $\varphi$ and $|31\rangle,|32\rangle$ for the parameter $\gamma$.

Now, if we apply the value of $x$ to the top -left beam splitter, $2 x$ to the top-right, the value $r$ to the bottom - left and $s$ to the bottom right (see fig. 4 , and the resulting probability distributions will be multiplied by 4 we obtain the final function in the form:

$$
\mu(x)=\frac{1+\cos (x+r)}{2}+\frac{1+\cos (2 x+s)}{2} .
$$

This function is the realization of QCoSamp made of two components.

To conclude the method we have to indicate what part of the whole current is a single QCoSamp Component and the method of addition of two or more component. Well, because of the nature of our system the components are not clearly separated. We can say, with a rough approximation, that the beam splitters applied on the output of MOS with phase shifters on one output designates the separate components, since using the phases of phase shifters we change the argument of the component. The addition is realized by entanglement propagation made by rail cross application of beam splitters

\footnotetext{
4 This is not normalized value, to obtain the proper probability one should account the normalization coefficients: $\frac{1}{2}$ from two last equations and $-\frac{1}{2}$ from the output state of MOS, which gives $\frac{1}{16}$ normalization factor for probability. It was omitted in this considerations for simplicity.

ditto

6 Three dimension qudits are called: qutrits
} 
Using more non-linear units and crossing theirs output as was described above, one can construct the setups for more components.

\section{Discussion and Conclusions}

In current work we showed how to build an optical setup to create single component for QCoSamp operator and how to add two or more components to create a series described in our previous work [4]. Nonetheless the described setup offers more forms of an output. Looking once again on the table with probabilities (fig. 4 right-bottom table), we see that there are encoded other functions of $x, r, s$, e.g. $4 \cos ^{2}(x-y)$, when we measure first and third ququartit and we take the probability for basis states: $|11\rangle$. This creates the opportunity to generate other forms of series that can be used for optimization algorithms.

This work opens further researches in several areas:

- The detection of temporal correlation of 4 and more photons in two polarization states. For the purpose of our setup there is a need of specific detection that will point the ququartits states. So for example the state $|00\rangle$ has to be detected by observing the remaining part of the system. On the other hand the states with two photon on the same rail with the same polarization $(\mathrm{e} . \mathrm{g},|20\rangle)$ has to be rejected, since we don't use it for computations.

- The implementation of distributed phase encoding [4,9], which is needed for implementation of quantum algorithms like quantum summations [18] or optimization [19].

- The implementation of amplitude amplification algorithm [10,11], which the core of optimization methods that can be applied in QCoSamp.

\section{Author Contributions}

Conceptualization, K.W; methodology, K.W; validation, A.M and J.G.; formal analysis, K.Wereszczyński and A.M.; investigation, K.Wereszczyński and M.P.; writing-original draft preparation, K.Wereszczyński; writing-review and editing, K.W., A.M., M.P. and J.G.; visualization, J.G.; supervision, K.W.; project administration, A.M.; funding acquisition, M.P.. All authors have read and agreed to the published version of the manuscript.

\section{Funding}

This research was funded by the research project for young scientists (RAU-6, 2020 and RAU-6, 2021) of the Silesian University of Technology.

\section{Conflict of Interests}

The authors declare no conflict of interest.

\section{References}

1. Knill, E.; Laflamme, R.; Milburn, G.J. A scheme for efficient quantum computation with linear optics. Nature 2001, 409, 46-52. doi:10.1038/35051009.

2. Bourassa, J.E.; Alexander, R.N.; Vasmer, M.; Patil, A.; Tzitrin, I.; Matsuura, T.; Su, D.; Baragiola, B.Q.; Guha, S.; Dauphinais, G.; Sabapathy, K.K.; Menicucci, N.C.; Dhand, I. Blueprint for a Scalable Photonic Fault-Tolerant Quantum Computer. Quantum 2021, 5, 392. arXiv: 2010.02905 version: 2, doi:10.22331/q-2021-02-04-392.

3. Mirrahimi, M.; Leghtas, Z.; Albert, V.V.; Touzard, S.; Schoelkopf, R.J.; Jiang, L.; Devoret, M.H. Dynamically protected catqubits: a new paradigm for universal quantum computation. New Journal of Physics 2014, 16, 045014. arXiv: 1312.2017, doi:10.1088/1367-2630/16/4/045014.

4. Wereszczyński, K.; Michalczuk, A.; Pęszor, D.; Paszkuta, M.; Cyran, K.; Polański, A. Cosine series quantum sampling method with applications in signal and image processing. arXiv:2011.12738 [quant-ph] 2020, [2011.12738].

5. Couteau, C. Spontaneous parametric down-conversion. Contemporary Physics 2018, 59, 291-304. doi:10.1080/00107514.2018.1488463.

6. Magnitskiy, S.; Frolovtsev, D.; Firsov, V.; Gostev, P.; Protsenko, I.; Saygin, M. A SPDC-Based Source of Entangled Photons and its Characterization. Journal of Russian Laser Research 2015, 36, 618-629. doi:10.1007/s10946-015-9540-x. 
7. Wang, X.L.; Chen, L.K.; Li, W.; Huang, H.L.; Liu, C.; Chen, C.; Luo, Y.H.; Su, Z.E.; Wu, D.; Li, Z.D.; Lu, H.; Hu, Y.; Jiang, X.; Peng, C.Z.; Li, L.; Liu, N.L.; Chen, Y.A.; Lu, C.Y.; Pan, J.W. Experimental Ten-Photon Entanglement. Phys. Rev. Lett. $2016,117,210502$. doi:10.1103/PhysRevLett.117.210502.

8. Zhong, H.S.; Li, Y.; Li, W.; Peng, L.C.; Su, Z.E.; Hu, Y.; He, Y.M.; Ding, X.; Zhang, W.; Li, H.; Zhang, L.; Wang, Z.; You, L.; Wang, X.L.; Jiang, X.; Li, L.; Chen, Y.A.; Liu, N.L.; Lu, C.Y.; Pan, J.W. 12-Photon Entanglement and Scalable Scattershot Boson Sampling with Optimal Entangled-Photon Pairs from Parametric Down-Conversion. Phys. Rev. Lett. 2018, 121 , 250505. doi:10.1103/PhysRevLett.121.250505.

9. Ruiz-Perez, L.; Garcia-Escartin, J.C. Quantum arithmetic with the quantum Fourier transform. 16. doi:10.1007/s11128-017-1603-1.

10. Grover, L.K. A fast quantum mechanical algorithm for database search. Proceedings of the twenty-eighth annual ACM symposium on Theory of computing - STOC '96; ACM Press: Philadelphia, Pennsylvania, United States, 1996; pp. $212-219$. doi:10.1145/237814.237866.

11. Brassard, G.; Hoyer, P. An Exact Quantum Polynomial-Time Algorithm for Simon's Problem. Proceedings of the Fifth Israeli Symposium on Theory of Computing and Systems 1997, pp. 12-23. arXiv: quant-ph/9704027, doi:10.1109/ISTCS.1997.595153.

12. Kok, P.; Munro, W.J.; Nemoto, K.; Ralph, T.C.; Dowling, J.P.; Milburn, G.J. Review article: Linear optical quantum computing. Reviews of Modern Physics 2007, 79, 135-174, [quant-ph/0512071]. doi:10.1103/RevModPhys.79.135.

13. Hong, C.K.; Ou, Z.Y.; Mandel, L. Measurement of subpicosecond time intervals between two photons by interference. Phys. Rev. Lett. 1987, 59, 2044-2046. Publisher: American Physical Society, doi:10.1103/PhysRevLett.59.2044.

14. Knill, E. Bounds on the probability of success of postselected nonlinear sign shifts implemented with linear optics. Phys. Rev. A 2003, 68, 064303. doi:10.1103/PhysRevA.68.064303.

15. Ji, Y.; Chung, Y.; Sprinzak, D.; Heiblum, M.; Mahalu, D.; Shtrikman, H. An electronic Mach-Zehnder interferometer. Nature 2003, 422, 415-418. doi:10.1038/nature01503.

16. Rarity, J.; Tapster, P.; Jakeman, E.; Larchuk, T.; Campos, R.; Teich, M.; Saleh, B. Two-photon interference in a Mach-Zehnder interferometer. Physical Review Letters 1990, 65, 1348-1351. doi:10.1103/PhysRevLett.65.1348.

17. Guo, X.; Meng, Z.; Li, J.; Yang, J.Z.; Aili, M.; Zhang, A.N. The interference properties of single-photon vortex beams in Mach-Zehnder interferometer. Applied Physics Letters 2021, 119, 011103. doi:10.1063/5.0055428.

18. Heinrich, S.; Novak, E. On a problem in quantum summation. Journal of Complexity 2003, 19, 1 - 18. doi:https://doi.org/10.1016/S0885064X(02)00003-1.

19. Harrow, A.W.; Hassidim, A.; Lloyd, S. Quantum Algorithm for Linear Systems of Equations. Physical Review Letters 2009, 103, 150502. doi:10.1103/PhysRevLett.103.150502. 